PROCEEDINGS OF THE

AMERICAN MATHEMATICAL SOCIETY

Volume 133, Number 3, Pages 671-675

S 0002-9939(04)07645-2

Article electronically published on August 24, 2004

\title{
HALL SUBGROUPS OF $M$-GROUPS NEED NOT BE $M$-GROUPS
}

\author{
HIROSHI FUKUSHIMA
}

(Communicated by Jonathan I. Hall)

\begin{abstract}
In this paper, we shall give examples of $M$-groups that have Hall subgroups that are not $M$-groups.
\end{abstract}

\section{INTRODUCTION}

A character of a finite group $G$ is monomial if it is induced from a linear character of a subgroup of $G$. A group $G$ is an $M$-group if all its complex irreducible characters (the set $\operatorname{Irr}(G)$ ) are monomial.

In the 1960s, Dornhoff [3] proved that normal Hall subgroups of $M$-groups are $M$-groups, and conjectured that normal subgroups of $M$-groups are $M$-groups. In the early 1970s, Dade [1] and van der Waall [6] independently showed that normal subgroups of $M$-groups need not be $M$-groups. In [5], Isaacs asked whether Hall subgroups of $M$-groups need be $M$-groups. In this paper, we shall give examples of $M$-groups that have Hall subgroups that are not $M$-groups.

Let $N \triangleleft G$ and $\theta \in \operatorname{Irr}(N)$. We write $C_{G}(\theta)$ to denote the stabilizer of $\theta$ in $G$. We also write $\operatorname{Irr}(G \mid \theta)=\left\{\chi \in \operatorname{Irr}(G) \mid\left[\chi_{N}, \theta\right] \neq 0\right\}$.

\section{Preliminary Lemmas}

We begin with some preliminary lemmas.

Lemma 2.1. Let $E$ be an extra-special p-group, let $\sigma$ be an automorphism of $E$ of order $q$ where $q \neq p$ is a prime, and let $G=E\langle\sigma\rangle$. If $\sigma$ acts irreducibly on $E / Z(E)$, then $G$ is not an M-group.

Proof. We know that $|E|=p^{2 n+1}$ for some positive integer $n$. This implies that the nontrivial irreducible $p$-modules for $\sigma$ must have dimension $2 n$, and thus, $\sigma$ must centralize $Z(E)$. For any nonlinear character $\psi \in \operatorname{Irr}(E)$, we see that $\psi(1)=p^{n}$ and $\psi$ is invariant under the action of $\sigma$. By Corollary 6.28 of [4], $\psi$ has an extension $\chi \in \operatorname{Irr}(G)$.

We claim that $\chi$ is not monomial. Suppose $\chi$ were monomial. Then there would be a subgroup $H \subseteq G$ and a linear character $\lambda \in \operatorname{Irr}(H)$ so that $\lambda^{H}=\chi$. We see that $|G: H|=\chi(1)=p^{n}$. It follows that $|H|=p^{n+1} q$, and so by conjugating, we may assume that $\sigma \in H$. This implies that $H \cap E / Z(E)$ is a $\sigma$-submodule of $E / Z(E)$, but this is a contradiction, since $Z(E) \subset H \cap E \subset E$ and $E / Z(E)$ is

Received by the editors June 23, 2003 and, in revised form, October 29, 2003.

2000 Mathematics Subject Classification. Primary 20C15.

(C)2004 American Mathematical Society Reverts to public domain 28 years from publication 
irreducible under the action of $\sigma$. Therefore, we conclude that $\chi$ is not monomial, and hence, $G$ is not an $M$-group.

Lemma 2.2. Let $N$ be a normal subgroup of $G$, and let $\varphi$ be a linear character of $N$. Write $T$ for the stabilizer of $\varphi$ in $G$, and assume that $\varphi$ extends to $\hat{\varphi} \in \operatorname{Irr}(T)$. Given a character $\eta \in \operatorname{Irr}(T / N)$, the character $(\hat{\varphi} \eta)^{G}$ is monomial if and only if $\eta$ is monomial. Furthermore, every character in $\operatorname{Irr}(G \mid \varphi)$ is monomial if and only if $T / N$ is an $M$-group.

Proof. By Gallagher's theorem, we know that $\hat{\varphi} \eta \in \operatorname{Irr}(T \mid \varphi)$, and by Clifford's theorem, $\chi=(\hat{\varphi} \eta)^{G}$ is irreducible. Using Lemma 4.1 of [2] (or Problem 6.11 of [4]), we see that $\chi$ is monomial if and only if $\hat{\varphi} \eta$ is monomial. Suppose $S$ is a subgroup of $T$ and $\sigma \in \operatorname{Irr}(S)$ so that $\sigma^{T}=\eta$. It is known that $\left(\hat{\varphi}_{S} \sigma\right)^{T}=\hat{\varphi} \eta$ (see Problem 5.3 of [4]). It follows that $\hat{\varphi} \eta$ is monomial if and only if $\eta$ is monomial. The last conclusion is an immediate consequence of the previous one, and this proves the lemma.

Lemma 2.3. Let $p$ be an odd prime so that 3 divides $p+1$. Let $E$ be an extra-special p-group of order $p^{5}$ and exponent $p$. Then $E$ has an automorphism $\sigma$ of order 3 with centralizer $Z(E)$, and $E$ has a maximal abelian subgroup $A$ that is normal in $E$ and $\sigma$-invariant. If $G$ is the semi-direct product $E\langle\sigma\rangle$, then $G$ is an $M$-group.

Proof. We know that 3 divides $p^{2}-1$. We can view $E$ as the central product of $E_{1}$ and $E_{2}$ where $E_{1}$ and $E_{2}$ are both extra-special groups of order $p^{3}$ and exponent $p$. Since 3 divides $p^{2}-1$, it follows that $E_{1}$ and $E_{2}$ each have an automorphism of order 3 with each having centralizer $Z=Z(E)$. Applying these to the central product, we get the automorphism $\sigma$ of $E$ with order 3 and centralizer $Z$. We let $T=\langle\sigma\rangle$, and we note that $C_{E}(T)=Z$. It suffices to find an abelian $T$-invariant subgroup $A \subseteq E$ of index $p^{2}$. (Note that this will prove that $G$ is an $M$-group.)

Let $U / Z$ be an irreducible $T$-submodule of $E / Z$. Then $U / Z$ has order $p^{2}$ since 3 does not divide $p-1$. If $U$ is abelian, then we are done, so we assume $U$ is nonabelian. Let $V=C_{E}(U)$. Then $U \cap V=Z$, and $E / Z$ is the direct sum of the irreducible modules $U / Z$ and $V / Z$. Note that $V$ must be nonabelian.

Take $x$ to be an element of $U$ that does not lie in $Z$, and write $y=x^{\sigma}$. Then $x$ and $y$ generate $U$, so they do not commute. Let $z=[x, y]$, and observe that $Z=\langle z\rangle$. Then $y^{\sigma} \in x^{-1} y^{-1} Z$.

Let $r$ be an element of $V$ that does not lie in $Z$, and write $s=r^{\sigma}$. We see that $r$ and $s$ generate $V$, so they do not commute, and hence, $[r, s]$ is a nonidentity element of $Z$. We see that $s^{\sigma} \in r^{-1} s^{-1} Z$. Suppose that $[r, s]=z^{-1}$. We observe that $(x r)^{\sigma}=y s$, and we compute $[x r, y s]=[x, y][r, s]=z z^{-1}=1$. Also, $(y s)^{\sigma} \in$ $\left(x^{-1} y^{-1}\right)\left(r^{-1} s^{-1}\right) Z=(x r)^{-1}(y s)^{-1} Z \subseteq\langle x r, y s\rangle$. We conclude that $\langle x r, y s\rangle$ is a $\sigma$-invariant abelian subgroup of $E$ of index $p^{2}$, and we will be done.

The idea is to choose $r$ properly. We pick any element $v \in V-Z$, and let $w=v^{\sigma}$. Note that $w^{\sigma} \in v^{-1} w^{-1} Z$. We know that $[v, w]=z^{a}$ for some integer $a$ with $1 \leq a \leq p-1$. We consider elements of the form $v^{i} w^{j}$, and we see that $\left(v^{i} w^{j}\right)^{\sigma} Z=w^{i} v^{-j} w^{-j} Z=v^{-j} w^{i-j} Z$. It follows that

$$
\left[v^{i} w^{j},\left(v^{i} w^{j}\right)^{\sigma}\right]=\left[v^{i} w^{j}, v^{-j} w^{i-j}\right]=\left(z^{a}\right)^{i(i-j)-j(-j)}=z^{a\left(i^{2}-i j+j^{2}\right)} .
$$

We need to show that as $i$ and $j$ vary over $Z / p Z$, the quantity $i^{2}-i j+j^{2}$ takes on all values in $Z / p Z$. 
For any value $b \in Z / p Z$, we consider the equation $i^{2}-i j+j^{2}=b$. We take the equation $i^{2}-i j+j^{2}-b=0$, and we solve for $i$. By the quadratic formula, we can do this if the discriminant is a square. The discriminant is $j^{2}-4\left(j^{2}-b\right)=4 b-3 j^{2}$. We want to find $k$ so that $4 b-j^{2}=k^{2}$. As $j$ and $k$ vary through the $p$ possible values in $Z / p Z$, we see that $4 b-3 j^{2}$ and $k^{2}$ each take on $(p+1) / 2$ different values. Since there are only $p$ possible values in $Z / p Z$, there must be an overlap between these two sets. We now fix $b$ so that $a b=-1$ modulo $p$. The work we have just done shows that we can find $i$ and $j$ so that $i^{2}-i j+j^{2}=b$ modulo $p$. We take $r=v^{i} w^{j}$, and we see that $s=r^{\sigma}$. The work we did earlier shows that $[r, s]=z^{a\left(i^{2}-i j+j^{2}\right)}=z^{a b}=z^{-1}$. We now conclude that $E$ has a normal $\sigma$-invariant subgroup of index $p^{2}$, so the lemma is proved.

\section{The COnstruction}

We suppose that $p$ and $q$ are distinct odd primes so that $p$ divides $q-1$ and 3 divides $p+1$. Then $q=1+p k$ for some integer $k$. Hence $\left(q^{p}-1\right) /(q-1)=$ $1+q+\cdots+q^{p-1} \equiv 1+(1+p k)+\cdots+(1+(p-1) p k)=p+\left((p-1) p^{2} k / 2\right)\left(\bmod p^{2}\right)$. Thus $\left(q^{p}-1\right) /(q-1)=p r$, where $r \equiv 1(\bmod p)$. In particular, $(p, r)=1$. Next we claim that 3 does not divide $r$. It is known that the ged of $q-1$ and $\left(q^{p}-1\right) /(q-1)$ must divide $p$, so if 3 divides $q-1$, then 3 will not divide $\left(q^{p}-1\right) /(q-1)$. On the other hand, we know that the order of $q$ modulo 3 must divide 2 , so if 3 does not divide $q-1$, then the order of $q$ modulo 3 is 2 . Since 2 does not divide $p$, it cannot be that $q^{p}$ is congruent to 1 modulo 3 , so 3 does not divide $\left(q^{p}-1\right) /(q-1)$.

We mention that there exist pairs of primes with the properties mentioned in the previous paragraph. One such pair of primes is $p=5$ and $q=11$. Observe that $\left(11^{5}-1\right) /(11-1)=5 \cdot 3221$.

Let $F$ be the finite field of order $q^{p}$. Take $V$ to be the additive group of $F$, so $V$ is an elementary abelian $q$-group. Let $N$ be the subgroup of of order $\left(q^{p}-1\right) /(q-1)=$ $p r$ in the multiplicative group of $F$. Multiplication in $F$ provides a natural action of $N$ on $V$ via automorphisms. The orbits in this action correspond to the cosets of the subgroup of order $q-1$ in the multiplicative group of $F$. Fix $s, t \in N$ so that $o(s)=p$ and $o(t)=r$, and note that $N=\langle s t\rangle$. Let $a$ be a generator for the Galois group of $F$ over the field of order $q$ so that $a$ has order $p$. The Galois action provides a natural action for $a$ on $V$ and $N$. Note that the fixed field for $a$ is the field of order $q$, so each orbit of $N$ on $V$ is stabilized by $a$. Since $p$ divides $q-1$, it follows when $s$ is viewed as an element of $F$ that $s$ lies in the fixed field, so $a$ will centralize $s$.

Let $Q$ be an extra-special $p$-group of order $p^{5}$ and exponent $p$. Let $Z$ be the center of $Q$, and suppose that $Z$ is generated by $z$. We can fix the generators of $Q$ to be $x_{0}, y_{0}, x_{1}, y_{1}$ so that $x_{0}^{p}=y_{0}^{p}=x_{1}^{p}=y_{1}^{p}=z^{p}=1$ and $\left[x_{0}, y_{0}\right]=\left[x_{1}, y_{1}\right]=z$. We define $Q_{0}=\left\langle x_{0}, y_{0}\right\rangle$ and $Q_{1}=\left\langle x_{1}, y_{1}\right\rangle$. Let $K$ be an elementary abelian group of order $p^{2}$ that is generated by $x_{2}$ and $y_{2}$. It is not difficult to see that $E=Q \times K$ has an automorphism $\sigma$ of order 3 that centralizes $Z$ and is defined by

$$
x_{0}^{\sigma}=y_{0}, y_{0}^{\sigma}=x_{0}^{-1} y_{0}^{-1}, x_{1}^{\sigma}=y_{1}, y_{1}^{\sigma}=x_{1}^{-1} y_{1}^{-1}, x_{2}^{\sigma}=y_{2}, y_{2}^{\sigma}=x_{2}^{-1} y_{2}^{-1} .
$$

We define $M$ to be the semi-direct product arising from $\sigma$ acting on $E$.

We set $U=V\langle t\rangle$. We define an action of $Q$ on $U$ whose kernel is $Q_{0}$ by $u^{x_{1}}=u^{a}$ and $u^{y_{1}}=u^{s}$ for all $u \in U$. We define an action of $K$ on $U$ by $u^{x_{2}}=u^{a^{-1}}$ and $u^{y_{2}}=u^{s}$ for all $u \in U$. We set $U_{0}=U \times U^{\sigma} \times U^{\sigma^{2}}$. We define an action of $M$ 
on $U_{0}$ by $\left(u^{\sigma^{i}}\right)^{x}=u^{\left(\sigma^{i} x \sigma^{-i}\right) \sigma^{i}}$ for all $u \in U, x \in M$, and $i=1,2$. Our group $G$ is the resulting semi-direct product of $M$ acting on $U_{0}$. Observe that $|U|=q^{p} r$ and $|M|=p^{7} 3$, so $|G|=q^{3 p} r^{3} p^{7} 3$. Take $V_{0}=V \times V^{\sigma} \times V^{\sigma^{2}}$, and let $H$ be the semidirect product of $M$ acting on $V_{0}$. We see that $|H|=q^{3 p} p^{7} 3$ and $|G: H|=r^{3}$, so $H$ is a Hall subgroup of $G$. (Obviously, $q$ does not divide $r$, the choice of $p$ precludes $p$ from dividing $r$, and we showed that 3 does not divide $r$; so $\left(r^{3}, q^{3 p} p^{7} 3\right)=1$.) We will show that $G$ is an $M$-group and $H$ is not an $M$-group. Also, we take $L$ to be the semi-direct product of $M$ acting on $\langle t\rangle \times\left\langle t^{\sigma}\right\rangle \times\left\langle t^{\sigma^{2}}\right\rangle$. Observe that $L$ acts coprimely on $V_{0}$.

Lemma 3.1. $H$ is not an M-group.

Proof. Let $A=Q_{0}\left\langle x_{1} x_{2}, y_{1} y_{2}^{-1}\right\rangle$. It is not difficult to see that $A$ is the kernel of the action of $E$ on $\operatorname{Irr}(V)$. Furthermore, since $E / A$ is abelian, it must have a regular orbit in $\operatorname{Irr}(V)$. So we can find a character $\lambda \in \operatorname{Irr}(V)$ with $C_{E}(\lambda)=A$. Let $\varphi=\lambda \times \lambda^{\sigma} \times \lambda^{\sigma^{2}}$. We see that $C_{E}(\varphi)=A \cap A^{\sigma} \cap A^{\sigma^{2}}$. It follows that $Q_{0} \subseteq C_{E}(\varphi)$. Also, $A$ is not $\sigma$-invariant, so $\left|E: C_{E}(\varphi)\right|>|E: A|=p^{2}$. We obtain $\left|C_{E}(\varphi): Q_{0}\right|<p^{2}$, and we conclude that $C_{E}(\varphi)=Q_{0}$. Since $\sigma$ will stabilize $\varphi$, we have $C_{M}(\varphi)=Q_{0}\langle\sigma\rangle$, and $C_{M}(\varphi)$ is not an $M$-group by Lemma 2.1.

Let $T$ be the stabilizer of $\varphi$ in $H$. It follows that $T=V_{0} C_{M}(\varphi)$. Since $\left(\left|V_{0}\right|, \mid T\right.$ : $\left.V_{0} \mid\right)=1$, we know $\varphi$ extends to $\hat{\varphi} \in \operatorname{Irr}(T)$. Since $T / V_{0} \cong C_{M}(\varphi)$, we can find a character $\eta \in \operatorname{Irr}\left(T / V_{0}\right)$ that is not monomial. We know that $(\hat{\varphi} \eta)^{H}$ is irreducible, and it is not monomial by Lemma 2.2. Therefore, $H$ is not an $M$-group.

Lemma 3.2. $G$ is an $M$-group.

Proof. Using Lemma 2.3, it is not difficult to show that $M \cong G / U_{0}$ is an $M$-group. To show $G$ is an $M$-group, it suffices to show that every character in $\operatorname{Irr}(G)$ whose kernel does not contain $U_{0}$ is monomial.

Suppose $\chi \in \operatorname{Irr}(G)$ and $U_{0}$ is not contained in $\operatorname{Ker}(\chi)$. For now, we will assume that $V_{0}$ is contained in $\operatorname{Ker}(\chi)$. Let $\varphi$ be an irreducible constituent of $\chi_{U_{0}}$, and notice that $\varphi \in \operatorname{Irr}\left(U_{0} / V_{0}\right)$. Let $T$ be the stabilizer of $\varphi$ in $G$, and observe that $\left(\left|U_{0}: V_{0}\right|,\left|T: U_{0}\right|\right)=1$, so $\varphi$ extends to $\hat{\varphi} \in \operatorname{Irr}(T)$. By Lemma 2.2, it suffices to prove that $T / U_{0}$ is an $M$-group. If 3 does not divide $\left|T: U_{0}\right|$, then $T / U_{0}$ is a $p$-group, and we are done. Thus, we may assume that 3 divides $\left|T: U_{0}\right|$, and by conjugating, we may assume that $\sigma \in T$. This implies that $\varphi=\nu \times \nu^{\sigma} \times \nu^{\sigma^{2}}$ for some character $\nu \in \operatorname{Irr}(U / V)$. Observe that $T=U_{0} C_{M}(\varphi)$ and $C_{M}(\varphi)=C_{E}(\varphi)\langle\sigma\rangle$. Furthermore, we have $C_{E}(\varphi)=C_{E}(\nu) \cap C_{E}(\nu)^{\sigma} \cap C_{E}(\nu)^{\sigma^{2}}=C_{E}(\nu) \cap C_{E}(\nu)^{\sigma}$. It is not difficult to see that $\left|E: C_{E}(\nu)\right|=p$ and $C_{E}(\nu) \cap C_{E}(\nu)^{\sigma}$ is an extra-special $p$-group of order $p^{5}$. Thus, $C_{M}(\varphi)$ is an $M$-group by Lemma 2.3 .

Finally, we assume that $V_{0}$ is not contained in the kernel of $\chi$. Let $\delta$ be an irreducible constituent of $\chi_{V_{0}}$. Let $S$ be the stabilizer of $\delta$ in $G$. Again, $\left(\left|V_{0}\right|, \mid S\right.$ : $\left.V_{0} \mid\right)=1$, so $\delta$ extends to $\hat{\delta} \in \operatorname{Irr}(T)$, and we see using Lemma 2.2 that it suffices to show that $S / V_{0}$ is an $M$-group. If 3 does not divide $\left|S: V_{0}\right|$, then $S /\left(S \cap U_{0}\right)$ is a $p$-group and $\left(S \cap U_{0}\right) / V_{0}$ is abelian. By Theorem 6.23 of [4], this will force $S / V_{0}$ to be an $M$-group. We suppose that 3 does not divide $\left|S: V_{0}\right|$, and by conjugating, we may assume that $\sigma \in S$. This implies that $\delta=\lambda \times \lambda^{\sigma} \times \lambda^{\sigma^{2}}$ for some nonprincipal character $\lambda \in \operatorname{Irr}(V)$.

We know that $\langle t\rangle$ acts Frobeniusly on $V$, so no nonidentity element in $\langle t\rangle$ will stabilize $\lambda$. It follows that $C_{\langle t\rangle E}(\lambda)$ is a $p$-subgroup, so we can find an element 
$h \in\langle t\rangle$ so that $A=C_{\langle t\rangle E}\left(\lambda^{h}\right) \subseteq E$. Recall that the orbits in $V$ under the action of $\langle t\rangle E$ have size $p r$. It is not difficult to see that the orbits in $V$ have the same size, so $|\langle t\rangle E: A|=p r$, and hence, $A$ has index $p$ in $E$.

Let $\delta^{\prime}=\lambda^{h} \times\left(\lambda^{\sigma}\right)^{h^{\sigma}} \times\left(\lambda^{\sigma^{2}}\right)^{h^{\sigma^{2}}}$ and observe that $\delta^{\prime}=\delta^{\left(h, h^{\sigma}, h^{\sigma^{2}}\right)}$. Observe that $C_{E}\left(\delta^{\prime}\right)=A \cap A^{\sigma} \cap A^{\sigma^{2}}=A \cap A^{\sigma}$. We note that $A$ is not $\sigma$-invariant so $A \cap A^{\sigma} \subset A$. On the other hand, $A$ is normal in $E$ of index $p$, so $A \cap A^{\sigma}$ will be normal in $E$ of index $p^{2}$, and $\left|A \cap A^{\sigma}\right|=p^{5}$. Since $K$ is not contained in $A$, we have $K \times Q_{0} \neq A \cap A^{\sigma}$, and we conclude that $C_{E}\left(\delta^{\prime}\right)=A \cap A^{\sigma}$ is an extra-special group of order $p^{5}$. Now, $C_{M}\left(\delta^{\prime}\right)=C_{L}\left(\delta^{\prime}\right)$ is a conjugate of $C_{L}(\delta)$ that lies in $M$, so $C_{M}\left(\delta^{\prime}\right)=C_{E}\left(\delta^{\prime}\right)\langle\sigma\rangle$, and $C_{M}\left(\delta^{\prime}\right)$ is an $M$-group by Lemma 2.3. It follows that $S / V_{0} \cong C_{M}(\delta)$ is an $M$-group. This proves the theorem.

\section{Another construction}

We observe that Lemma 2.3 is still true if $E$ is replaced by a central product of two quaternion groups of order 8. We can change the construction in Section 3 by taking $p=2$ and $q$ to be an odd prime so that $q+1=2 r$ where $r$ is relatively prime to 6 . (The first such prime $q=13$.) We take $Q$ to be the central product of two quaternion groups of order 8 , and we make the appropriate changes in the generators of $Q$. The rest of the argument will go through for this construction.

\section{ACKNOWLEDGMENTS}

I wish to thank Mark L. Lewis for his many helpful suggestions, both for revising my presentation and generalizing the example. I also wish to thank Masayuki Wajima for providing me his preprint.

\section{REFERENCES}

[1] E.C.Dade. Normal subgroups of $M$-groups need not be $M$-groups. Math. Z. 133(1973), 313-317. MR 0325748 (48:4094)

[2] E.C.Dade. Monomial characters and normal subgroups. Math. Z. 178(1981), 401-420. MR $0635210(83 \mathrm{e}: 20014)$

[3] L.Dornhoff. $M$-groups and 2-groups. Math. Z. 100(1967), 226-256. MR 0217174 (36:265)

[4] I.M.Isaacs. Character theory of finite groups. Academic Press, San Diego, California, 1976. MR 0460423 (57:417)

[5] I.M. Isaacs, Primitive characters, normal subgroups, and $M$-groups, Math. Z. 177(1981), 267284. MR 0612879 (82f:20026)

[6] R.W.Van der Waall. On the embedding of minimal non- $M$-groups. Indag. Math. 36(1974), 157-167. MR 0352237 (50:4724)

Department of Mathematics, Faculty of Education, Gunma University Maebashi, GunMa 371-8510, JAPAN

E-mail address: fukusima@edu.gunma-u.ac.jp 